\title{
Memo-VR: Exercício Cognitivo para Idosos Utilizando Realidade Virtual e Interface com as Mãos
}

\author{
Flávio M. de Farias ${ }^{1,2}$, Eyre B. B. Montevecchi ${ }^{1,2}$, José Raphael Bokehi ${ }^{2}$, \\ Rosimere F. Santana ${ }^{3}$, Debora C. Muchaluat-Saade ${ }^{1,2}$ \\ ${ }^{1}$ Laboratório MídiaCom, ${ }^{2}$ Instituto de Computação \\ ${ }^{3}$ Escola de Enfermagem \\ Universidade Federal Fluminense (UFF) \\ \{flavio.farias, eyrebrasil, debora\}@midiacom.uff.br \\ jraphael@ic.uff.br, rosifesa@gmail.com
}

\begin{abstract}
Electronic games can be used as a support tool for treating depression, anxiety, and depression, as well as stimulating the brain through cognitive exercises. Virtual Reality (VR) can enhance the emotional engagement and immersion level in those exercises. Thus, this article proposes a novel cognitive exercise, called Memo-VR, which uses VR combined with interaction techniques, such as scanning and virtualization of the user's hands, in a version of the classic Concentration game. The tests with the elderly indicated as preliminary results good usability.
\end{abstract}

Resumo. Jogos eletrônicos têm sido aplicados como ferramentas de suporte a tratamentos para demência, ansiedade e depressão, assim como também podem ser utilizados para estimular o cérebro, na forma de exercícios cognitivos. Esses exercícios podem ser potencializados pelo sentimento de pertencimento e aumento do nível de imersão oferecido pela Realidade Virtual (VR). Desta forma, este artigo propõe um novo exercício cognitivo, chamado Memo-VR, que usa VR combinada a técnicas de interação como o escaneamento e virtualização das mãos do usuário, em uma versão do clássico jogo da memória. O jogo foi testado com um grupo de idosos, e embora preliminares, os resultados indicam uma boa usabilidade.

\section{Introdução}

À medida que ocorre o envelhecimento, as capacidades cerebrais e cognitivas são afetadas, de forma que atividades básicas podem ser prejudicadas, tais como lembrar senhas, lembrar onde um determinado documento se encontra, ou não conseguir usar as chaves de casa devido a problemas de coordenação motora [Oei and Patterson 2013].

Os jogos eletrônicos (videogames) são uma forma de diversão e lazer muito difundida atualmente e, assim como outras novas mídias, podem auxiliar diversas especialidades profissionais [Oei and Patterson 2013]. Na área da saúde, jogos e gamificação têm sido aplicados como ferramentas de suporte a tratamentos tradicionais para demência, ansiedade e depressão por exemplo [Vazini Taher et al. 2015].

Videogames também têm sido usados em pesquisas relacionadas a trabalhos que exigem coordenação psíquica e exercícios de treinamento cerebral para resolução de pro- 
blemas de forma rápida [Nouchi et al. 2013]. Dentre diversas pesquisas, outros trabalhos usam jogos como abordagem para exercitar a memória do usuário, seja por meio de jogos da memória ou por exercícios cognitivos [De Paula et al. 2019, Matos et al. 2018, Oei and Patterson 2013].

Este artigo propõe um novo exercício cognitivo, chamado Memo-VR, que usa a tecnologia de Realidade Virtual (do inglês, Virtual Reality - VR) combinada a técnicas de interação como o escaneamento e virtualização das mãos do usuário, em uma versão do clássico jogo da memória. Como esta pesquisa encontra-se em desenvolvimento, são apresentados resultados preliminares de testes com usuários idosos.

O restante do texto está estruturado da seguinte forma. Na Seção 2, são discutidas pesquisas relacionadas a jogos cognitivos. A Seção 3 apresenta o jogo desenvolvido Memo-VR e na Seção 4 são discutidos os testes e resultados preliminares. A Seção 5 conclui o artigo e aponta trabalhos futuros.

\section{Trabalhos Relacionados}

Dentre os diversos jogos eletrônicos para tratamento de queixa de perda de memória e/ou cognição irregular, o Jogo do Stroop [De Paula et al. 2019] e o MemoGinga [Matos et al. 2018] utilizam a plataforma de TV digital brasileira.

O Jogo do Stroop foi desenvolvido com o objetivo de treinar a capacidade de atenção seletiva dos idosos por meio da exploração do efeito Stroop ${ }^{1}$ : há uma interferência no tempo de reação provocada no cérebro ao apresentar informações conflitantes a respeito de um objeto. Por exemplo, nesse jogo, pode ser exibida na tela a palavra "verde" em cor vermelha, questionando qual palavra está escrita.

O MemoGinga é um jogo que tem como objetivo atuar na prevenção de problemas cognitivos relacionados a memória, especificamente a de curto prazo. O jogo apresenta pares de figuras geométricas coloridas (e.g. triângulos, quadrados, estrelas) em uma tela para memorização. Na próxima tela, é perguntado ao jogador se uma determinada figura foi apresentada anteriormente.

Os Jogos da Memória online dos sites Racha $\mathrm{Cuca}^{2}$ e Geniol ${ }^{3}$ são muito semelhantes ao Memo-VR, apresentado neste artigo, nos quesitos regras, recompensas e gamificação, mas com a vantagem de maior simplicidade, por serem jogos executados em navegadores web com a interação realizada somente com mouse. Porém, como desvantagem, não proporcionam a sensação de pertencimento e imersão que a realidade virtual permite. Esse tipo de jogo busca trabalhar com a memória de curto prazo e é de fácil assimilação.

Pietrzak et al. [Pietrzak et al. 2014] discute diferentes métodos de reabilitação usando VR para pessoas com déficits em funções motoras e cognitivas, sensações e emoções advindos de trauma crânio-encefálico (TCE). As soluções apresentadas mostram melhorias principalmente nos níveis de atenção, memória espacial e operacional e

\footnotetext{
${ }^{1}$ É uma demonstração de interferência no tempo de reação do cérebro quando exposto a uma discordância entre leitura e visualização de cores [De Paula et al. 2019].

${ }^{2}$ https://rachacuca.com.br/passatempos/jogo-da-memoria/

${ }^{3}$ https://www.geniol.com.br/passatempos/jogo-da-memoria
} 
tarefas de aprendizado visual. O uso de VR também é observado como forma de prover reabilitação de maneira mais acessível a lugares limitados financeira e geograficamente.

É muito comum que idosos tenham queixas relacionadas a falta de memória e dificuldades cognitivas. Em Barnes et al. [Barnes et al. 2007], é demonstrado que idosos com pontuação alta em testes cognitivos possuem uma maior independência e melhor qualidade de vida que os demais testados.

O trabalho Glass et al. [Glass et al. 2013] argumenta que apesar de jogos eletrônicos não conseguirem resultados com alto nível de desenvolvimento cerebral ou benefícios duradouros, podem aumentar a velocidade de processamento perceptivo, devido a estímulos cerebrais originados das diversas novas informações ofertadas pela atividade.

Além de explorar a pesquisa de jogos cognitivos para idosos, este trabalho tem como foco a utilização da Realidade Virtual, como ferramenta de imersão e isolamento do usuário das sensações externas que possam desviar a atenção das atividades sendo realizadas, e além disso, utilizar movimentos de escaneamento manuais para elevar o efeito de imersão e facilitar a interação com os objetos do jogo.

\section{O Jogo Memo-VR}

O Memo-VR baseia-se em um jogo clássico e popular de memorização e o objetivo é encontrar todos os pares de cartas do tabuleiro. Para isso, o usuário deve virar duas cartas de cada vez, revelando suas faces ao usuário. Quando essas forem iguais, são retiradas do tabuleiro, caso contrário, são viradas novamente escondendo o conteúdo e o erro é computado de forma transparente para o jogador.

A interface do jogo oferece as informações de número total de cartas no tabuleiro, número de erros e tempo corrente do jogo, além de ser ambientado num cenário virtual de sala de estar ensolarada, para transmitir uma sensação aconchegante ao jogador. Como penalização por erros do jogador, o jogo incrementa o número total de erros. Há três níveis de dificuldade baseados em quantidade de cartas no tabuleiro: cinco pares no nível fácil, sete no médio e dez no difícil. Após o término de cada partida são mostrados o placar, tempo de jogo e total de erros.

O jogo utiliza o headset de realidade virtual HTC Vive $e^{4}$, responsável por receber a virtualização advinda do computador e exibi-la ao usuário. Este dispositivo pode ser substituído por equipamentos semelhantes. O outro equipamento é o Leap Motion Controler ${ }^{5}$, um dispositivo fixado a frente do headset, que é responsável por escanear as mãos do usuário, permitindo sua visualização no ambiente virtual. Com o Leap Motion, o usuário pode interagir com o jogo por meio das suas próprias mãos, sem precisar segurar controles físicos convencionais. O jogo foi desenvolvido utilizando a engine de desenvolvimento Unity $^{6}$, um ferramenta de desenvolvimento de jogos digitais com suporte a realidade virtual, compatível com o sistema operacional Windows 10, e que possui licença de uso não comercial gratuita.

\footnotetext{
${ }^{4}$ https://www.vive.com/us/

${ }^{5}$ https://www.ultraleap.com/

${ }^{6}$ https://unity.com/pt
} 


\section{Testes e Resultados Preliminares}

Até o momento, foram realizados testes com sete usuárias no mês de dezembro de 2019. Todas eram idosas do sexo feminino com idade entre 61 a 85 anos, com grau de escolaridade que varia do ensino fundamental até a pós-graduação. Todas as usuárias participam de um projeto para informatização da terceira idade. Todas possuíam algum contato com equipamentos de informática, porém, somente uma delas já havia utilizado equipamento de realidade virtual anteriormente. Quase a metade delas $(42,9 \%)$ possui queixas de falta de memória, mas nenhuma possui distúrbio de memória diagnosticado por médico.

Devido ao público-alvo (i.e., idosos), os testes foram realizados com as jogadoras sentadas, para diminuir o desconforto e aumentar o nível e segurança do experimento como ilustrado na Figura 1(a). Com o suporte de um acompanhante ao lado, o jogo era monitorado e exibido em tela adicional em tempo real, como ilustrado na Figura 1(b). Todos os testes seguiram as regras descritas e aprovadas pelo conselho de ética da Universidade Federal Fluminense (UFF), com número de parecer 250.132.

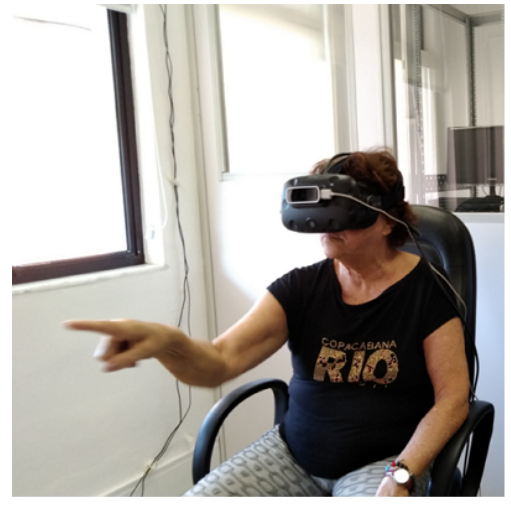

(a)

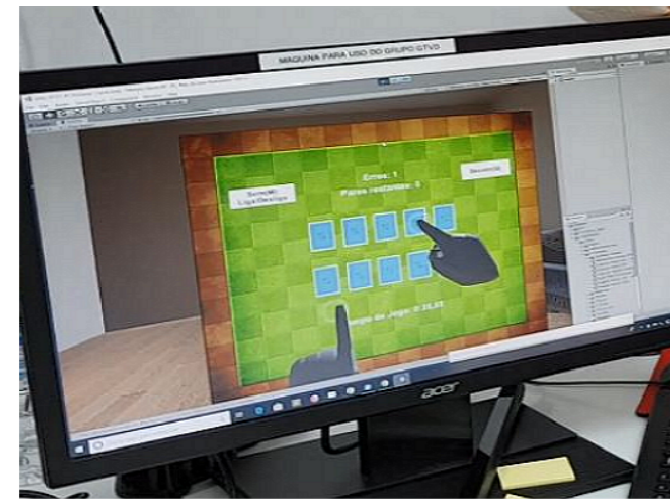

(b)

Figura 1. Usuária interagindo com o Memo-VR

Todas os usuárias tiveram tempo livre de jogo até que se sentissem confortáveis com o ambiente e ferramentas usadas (média de 90 segundos). Em seguida, cada uma delas jogava nos níveis fácil, médio e difícil, e tiveram seus resultados registrados. Depois dos testes, eram encaminhadas para responder um formulário contendo: perguntas sobre idade, grau de escolaridade, queixa de perda de memória; questionário System Usability Scale - SUS [outros Brooke 1996]; e questionário de experiência do usuário baseado no trabalho [Silva et al. 2018]. A seguir, os resultados preliminares obtidos são discutidos.

Na Tabela 1, é possível observar o crescimento do número de erros e média de tempo para a realização das tarefas com os respectivos desvios-padrão (Dp). Já na Tabela 2, há um resumo com algumas das respostas dadas ao questionário de experiência e ao SUS. Ambos usam a escala de Likert [Likert 1974], que divide as respostas em uma escala de cinco pontos, indicando grau de discordância ou concordância a respeito de uma afirmação. Para o questionário SUS, foi obtida uma pontuação média de 83 pontos, o que significa uma classificação nível $\mathrm{B}$, ou seja, excelente de acordo com Bangor, Kortum, e Miller [Bangor et al. 2009], que forneceram em seu trabalho uma escala de classificação absoluta para interpretação da pontuação média obtida com o SUS.

Das quase 30 questões apuradas, muitas possuem respostas pulverizadas na escala 
Tabela 1. Pontuação média e desvio padrão (DP) das respostas

\begin{tabular}{|c|r|r|r|r|r|c|}
\hline & \multicolumn{2}{|c|}{ Nível Fácil } & \multicolumn{2}{c|}{ Nível Médio } & \multicolumn{2}{c|}{ Nível Difícil } \\
\cline { 2 - 7 } & Média & DP & Média & DP & Média & DP \\
\hline Tempo (s) & 57,7 & 35,3 & 87,7 & 39,7 & 187,5 & 106,4 \\
\hline Número de Erros & 5 & 2,5 & 14 & 6,4 & 23 & 10,9 \\
\hline
\end{tabular}

Tabela 2. Resumo das respostas de algumas perguntas do Formulário de Experiência do Usuário e SUS

\begin{tabular}{|c|c|}
\hline Pergunta & Resumo (7 pessoas) \\
\hline Eu gostei de usar as mãos para interagir com o jogo & $\begin{array}{l}2 \text { Concordo parcialmente } \\
5 \text { Concordo fortemente }\end{array}$ \\
\hline $\begin{array}{l}\text { senti que a realidade virtual ajudou em uma melhor } \\
\text { memorização das cartas }\end{array}$ & $\begin{array}{l}1 \text { Discordo fortemente } \\
6 \text { Concordo fortemente }\end{array}$ \\
\hline Eu me senti parte do jogo (imersão) & $\begin{array}{l}1 \text { Discordo fortemente } \\
1 \text { Discordo parcialmente } \\
5 \text { Concordo fortemente }\end{array}$ \\
\hline Eu senti felicidade ao jogar & 7 Concordo fortemente \\
\hline Eu acho que gostaria de jogar esse jogo com frequência & 7 Concordo fortemente \\
\hline Eu achei o jogo complicado de jogar & $\begin{array}{l}1 \text { Discordo parcialmente } \\
6 \text { Discordo fortemente }\end{array}$ \\
\hline
\end{tabular}

de cinco pontos, o que indica a necessidade de testes com um número maior de usuários. Por isto, optou-se por mostrar somente as questões com número de resposta bem definido, que indicam que há uma boa aceitação do jogo pelo público idoso (Eu senti felicidade ao jogar) e que tem potencial para ser usado como exercício cognitivo com frequência (Eu acho que gostaria de jogar esse jogo com frequência). Outro ponto de destaque é no uso da interação com as mãos, em que todas as usuárias concordaram que gostaram de utilizar (Eu gostei de usar as mãos para interagir com o jogo).

\section{Considerações Finais}

Este artigo apresentou o jogo Memo-VR. Embora a pesquisa ainda esteja em andamento, a pontuação média de 83 pontos obtida para o questionário SUS indica que o jogo criado possui uma boa usabilidade. As usuárias também apontaram que se sentiram mais imersas e concentradas no ambiente de realidade virtual.

Como limitações deste trabalho, destaca-se o número pequeno de avaliações, o que não permite resultados totalmente conclusivos, mas planeja-se realizar mais testes em breve. Desta forma, poderá ser realizada uma avaliação mais efetiva, percebendo se 
existem correlações entre nível de escolaridade e quantidade de tempo ou acertos; idade e desempenho; e se a realidade virtual realmente auxilia na melhora da memorização e na concentração.

\section{Referências}

Bangor, A., Kortum, P., and Miller, J. (2009). Determining what individual SUS scores mean: Adding an adjective rating scale. Journal of usability studies, 4(3):114-123.

Barnes, D. E., Cauley, J. A., Lui, L. Y., Fink, H. A., McCulloch, C., Stone, K. L., and Yaffe, K. (2007). Women who maintain optimal cognitive function into old age. Journal of the American Geriatrics Society, 55(2):259-264.

De Paula, G. L., De Melo, L. F., Dos Santos, M. H., Seixas, F. L., Santana, R. F., and Muchaluat-Saade, D. (2019). Efeitos Sensoriais em Jogos Cognitivos para Idosos: Jogo do Stroop. In Anais Principais do XIX Simpósio Brasileiro de Computação Aplicada à Saúde, pages 187-198. SBC, Sociedade Brasileira de Computacao - SB.

Glass, B. D., Maddox, W. T., and Love, B. C. (2013). Real-Time Strategy Game Training: Emergence of a Cognitive Flexibility Trait. PLoS ONE, 8(8).

Likert, R. (1974). A method of constructing an attitude scale. Scaling: A sourcebook for behavioral scientists, pages 233-243.

Matos, L. F., Santos, M. H., Boechat, Y., Amorim, G., and Muchaluat-Saade, D. C. (2018). MemoGinga: Jogo Interativo para TV Digital como Exercício Cognitivo para Idosos. In Anais do Simpósio Brasileiro de Computação Aplicada à Saúde (SBCAS). Sociedade Brasileira de Computação - SBC.

Nouchi, R., Taki, Y., Takeuchi, H., Hashizume, H., Nozawa, T., Kambara, T., Sekiguchi, A., Miyauchi, C. M., Kotozaki, Y., Nouchi, H., et al. (2013). Brain training game boosts executive functions, working memory and processing speed in the young adults: a randomized controlled trial. PloS one, 8(2).

Oei, A. C. and Patterson, M. D. (2013). Enhancing Cognition with Video Games: A Multiple Game Training Study. PLoS ONE, 8(3).

outros Brooke, J. e. (1996). SUS-A quick and dirty usability scale. Avaliação de usabilidade na indústria, 189:4-7.

Pietrzak, E., Pullman, S., and McGuire, A. (2014). Using virtual reality and videogames for traumatic brain injury rehabilitation: a structured literature review. GAMES FOR HEALTH: Research, Development, and Clinical Applications, 3(4):202-214.

Silva, R. S., Carlos, C. A., Mol, A. M., and Ishitani, L. (2018). Avaliação da Experiência da Terceira Idade com Realidade Virtual na Área de Jogos Digitais para Smartphone. Art Des Track, pages 79-87.

Vazini Taher, A., Khalil Ahmadi, M., and Zamir, P. (2015). Effects of multi-sensory stimulation on cognition function, depression, anxiety and quality of life in elderly persons with dementia. International Journal of Sport Studies, 5(3):355-360. 